\title{
Higher Dietary Inflammatory Index Scores are Associated with Increased Odds of Diabetic Sensorimotor Polyneuropathy in Patients with Type 2 Diabetes Mellitus: A Case-Control Study
}

\section{Sara Asadi}

Tehran University of Medical Sciences

\section{Azadeh Aminianfar}

Tehran University of Medical Sciences

\section{Sasan Asadi}

Kurdistan University of Medical Science: Kurdistan University of Medical Sciences

Habib Yarizadeh

Tehran University of Medical Sciences

Mostafa Qorbani

Tehran University of Medical Sciences

Khadijeh Mirzaei ( $\nabla$ mirzaei_kh@tums.ac.ir)

Tehran University of Medical Sciences https://orcid.org/0000-0003-0231-0478

\section{Research}

Keywords: Inflammation, diabetes, diet, diabetic sensorimotor polyneuropathy.

Posted Date: September 16th, 2020

DOI: https://doi.org/10.21203/rs.3.rs-75457/v1

License: (c) (i) This work is licensed under a Creative Commons Attribution 4.0 International License.

Read Full License 


\section{Abstract}

Background: Diabetic sensorimotor polyneuropathy (DSPN) is a very common complication of diabetes. Dietary inflammatory score DII) has been associated with some diseases such as diabetes and cancers. Nonetheless, the association between DII and DSPN has not been explored. We aim to examine the association between DII and DSPN in a case-control study.

Method: 185 subjects with DSPN and 185 controls were selected in this case-control study. 168-item validated food frequency questionnaire (FFQ) was used to assed dietary intake. DII was calculated based on FFQ. DSPN was diagnosed by Toronto clinical neuropathy score. Anthropometric measurements, physical activity, glycemic indices were measured. Information on social demographic data was collected by questionnaires.

Result: After adjustment for age, sex, energy in model 1 ( $p$-trend< 0.001$)(\mathrm{OR}=0.25,95 \% \mathrm{Cl}=0.13-0.49)$, participants at the highest quartile of DII had increased OR for DSPN additionally adjusted for physical activity, education, smokers, FBS, A1C and economic status in model 2 (OR=0.27, 95\% Cl=0.13-0.57) ( $p$ trend $<0.001)$ strengthened the relationship. The association was not altered substantially by further adjustment for $\mathrm{BMI}$ in model $3(\mathrm{OR}=6.3,95 \% \mathrm{Cl}=2.7-14.6)$ ( $\mathrm{p}$-trend $<0.001)$.

Conclusion: Higher DII score was associated with increased risk of DSPN. Therefore, it is possible that a diet rich in anti-inflammatory nutrients and foods could improve and prevent DSPN.

\section{Introduction}

Diabetes mellitus (DM) is a very common metabolic disorder. It was estimated that 422 million people worldwide have been diagnosed with diabetes, which is the largest global epidemic of the 21st century[1]. Among the complications of diabetes, Diabetic sensorimotor polyneuropathy (DSPN) is very common. DSPN typically, is associated with increased risk of falling, pain and decreased quality of life[2, 3]. Moreover, DSPN is a major contributor to neuropathic ulcers and infection which can result in lower limb amputation[4]. Inflammation and oxidative stress that induced by chronic hyperglycemia and also mitochondrial dysfunction are three main important risk factors in the pathogenesis of DSPN [5].

In fact, regulating blood glucose through diet, exercise, or medication can play an important role in preventing diabetes and associated complications such as DSPN[6]. Diet plays an important role in the regulation of chronic inflammation with certain foods and nutrients [7]. A diet rich in red meat and saturated fat is associated with the activation of pro-inflammatory factors[8]. However, a whole grain diet group and fruits and vegetables group diet can inhibit the production of pro-inflammatory cytokines including Tumor Necrosis Factor-a (TNF-a), Interleukin-1 (IL-1) [9]. The dietary inflammatory index (DII) was designed to represent the total pro-inflammation of a whole diet [10]. The DII has been established in association with some inflammatory biomarkers $[11,12]$. The linkage between diet and inflammation and subsequent increase in the risk of developing Type 2 Diabetes Mellitus (T2DM) have been shown in previous studies. For example, Matthias B Schulze[13] showed that a diet that was high in sugar- 
sweetened soft drinks, refined grains, diet soft drinks, and processed meat but low in wine, coffee, cruciferous vegetables can result in increased inflammation and the risk of developing diabetes. Based on the results of a cross-sectional study, a pro-inflammatory diet can raise the risk of DM among adult Mexicans[14]. Among a cohort of Iranian adults, a cross-sectional study showed that DII had a positive association with post-load glucose (2 h-PG) [15]. A case-control study showed that patients with an antiinflammatory diet were at a decreased risk of pre-diabetes compared to patients with a pro-inflammatory diet [16]. Nonetheless, the association between DII and DSPN has not been explored. We aimed to examine the association between DII and DSPN in a case-control study.

\section{Methods}

\section{Study design and subjects}

This case-control study was carried out in the Diabetes Research Center, Endocrinology and Metabolism Clinical Sciences Institute, Kermanshah, Iran between September 2019 and April 2020. The present study included 185 cases with Non-Insulin-Dependent Diabetes Mellitus (NIDDM), who are aged 30-60 years old with Body Mass Index (BMI) between 25 to $39.9 \mathrm{~kg} / \mathrm{m}^{2}$ and diagnosed with DSPN caused by diabetes and 185 age- $( \pm 10)$, sex-matched controls. Patients were excluded based on the following criteria; 1$)$ those diagnosed with neuropathy due to other diseases, 2) patients with a history of diseases such as cancer, liver, kidney, autoimmune diseases, and inflammatory, thyroid and nervous and cardiovascular diseases, 3) pregnancy or lactation. Patients were also excluded if they had a particular diet during the recent last two months. The study protocol was approved by the Ethics Committee of Tehran University of Medical Science. TUMS.VCR.REC. 1399.269. All participants were required to complete written informed consent.

The following equation was used to calculate the sample size of the study:

$$
\mathrm{N}=\frac{\left(z_{1-\frac{\alpha}{2}}+z_{1-\beta}\right)^{2} p_{1}\left(1-p_{1}\right) p_{2}\left(1-p_{2}\right)}{\left(p_{2}-p_{1}\right)^{2}}
$$

Where $\mathrm{p}_{1}$ is the proportion exposed to DII in the control group while $\mathrm{p}_{2}$ is the proportion exposed to DII in the case group. Assuming $\alpha=0.05$ and $\beta=0.2$ and prevalence of diabetes in the first quartile or most antiinflammatory diet group and the fourth quartile or least anti-inflammatory diet group to be equal to 6.4 and 22.8 respectively[14], we needed 185 participants in each group.

\section{Dietary assessment and Dietary inflammatory Index calculation}

Dietary intakes were assessed using a valid and reliable semi-quantitative Food Frequency Questionnaire (FFQ) (168 food items, with standard serving sizes as commonly consumed by Iranians)[17]. One trained expert completed all FFQ questionnaires. All participants were asked about their average dietary intake on a daily, weekly, and monthly basis during the last year. Daily nutrients and energy intakes of foods and 
beverages were analyzed by Nutritionist software version 4(First Data Bank, San Bruno. CA) modified for Iranian foods.

DII was estimated by using FFQ and Shivappa et al [18]. In this regard, we included 29 parameters to calculate DII which were energy, carbohydrate, fat, protein, fiber, cholesterol, mono-unsaturated fatty acids (MUFAs), polyunsaturated fatty acids (PUFAs), saturated fats (SFAs), cobalamin, pyridoxine, folic acid, niacin, riboflavin, thiamin, vitamin A, C, D, E, b-carotene, zinc, selenium, magnesium, iron, caffeine, garlic, onion, pepper, and green/black tea. First, we calculated the Z score for all 29 parameters by the ratio of the standard global mean from the quantity of food parameters consumed by each participant to the global standard deviation. We obtained global mean and SD from Shivappa et al [18]. This value was converted to the percentile score. Then, the resulting of this value was multiplied by the effect score for all of the food parameters gained from Shivappa et al[18]. Eventually, the DII score from all the foods was summed to compute an overall DII score for each subject.

\section{Toronto clinical neuropathy score}

We used the Toronto Clinical Neuropathy Score (TCNS) to diagnose and compute the severity of neuropathy. It has been proved that TCNS is a valid and reliable questionnaire in Iran and other countries $[19,20]$. TCNS contains three sections; first, symptoms scores include the absence or presence of foot pain, numbness, tingling, and weakness in the feet, similar upper-limb symptoms, and ataxia (six points). Symptom scores rated as absence $=0$ or presence $=1$. Second, sensory test scores contain the absence or presence of pinprick, temperature, light touch, vibration, position sense that were performed at the first toe five points). Scores rated as absence $=0$ or presence $=1$. Third, Reflexes scores (knee reflexes, Ankle reflexes) which were rated as normal $=0$, reduced $=1$ or absence $=2$ (eight points). TCNS scores range from a minimum of 0 (without neuropathy) to a maximum of 19 points. Based on the result of TCNS, subjects were graded into classes of no neuropathy: 0-5; mild neuropathy: 6-8; moderate neuropathy: 911 ; and severe neuropathy $>12$. Clinical examinations were conducted by a neurologist and the results were recorded on a special form.

\section{Assessment of other variables}

A digital scale was used to determine the weight to the nearest $50 \mathrm{~g}$. height was measured to the nearest $0.5 \mathrm{~cm}$. Participants were asked to no wear shoes. BMI was computed through Weight $(\mathrm{kg})$ divided by height squared $(\mathrm{m} 2)$. A non-elastic tape with an accuracy of $0.5 \mathrm{~cm}$ was used to measure Waist circumference (WC) at a point midway between the iliac crest and lower rib margin. The short form of the International Physical Activity Questionnaire (IPAQ) was used to estimate physical activity during the last week. IPAQ is a validated self-reported seven-item measure of physical activity during the last week [21]. The anthropometric measurements were performed by a dietitian trained in anthropometry to reduce individual error.

We took fasted venous blood samples from all participants. We separated blood sample by centrifugation (at $3000 \mathrm{rpm}$ for $10 \mathrm{~min}$ at $4{ }^{\circ} \mathrm{C}$ ) to acquire serum. Then they were stored at $-80{ }^{\circ} \mathrm{C}$ until 
biochemical analyses performed. An auto-analyzer instrument (ERBA), using commercial kits (Pars Azmoon, Iran) were used to measure Fasting Blood Sugar(FBS) and Blood Sugar after 2 hours (Bs2hp). Also, using a high-Performance Liquid Chromatography (HPLC) (Advance scientific instrument, Germany) to estimate Glycated hemoglobin (HbA1c).

\section{Statistical Analysis}

Data were analyzed using SPSS 16 for Windows (SPSS Inc., Chicago, IL). First of all, quartile cut-off points for the DII score was defined. All patients were classified based on these cut-offs: q1<-1.03; q2 (-1.03)-(-0.01); q3 (-0.03)-(0.844); and q4 0.844<. To compare the means and distribution of continuous and categorical variables, independent-sample t-test and analysis of Chi-square test were used, respectively. Differences across quartiles of DII were compared using one-way ANOVA and Chi- square tests. Food and nutrient intakes were adjusted for age, sex and energy, except for dietary energy intake, which was only adjusted for age and sex using ANCOVA by quartiles of the DII. Using Binary logistic regression, to investigate associations between the DII and DSPN. Three regression models were used in our analysis. In model, it was adjusted for age, sex and energy intake. In model2, we adjusted further for physical activity, education, economic status, smoking, FBS, A1C, BS2hP. Moreover, in model 3 it was adjusted for BMI to perceive the relation when in-dependent of obesity. We defined the first quartile of DII as the reference category and odds ratios and $95 \% \mathrm{Cls}$ for the other quartiles were estimated. $\mathrm{P}$ values $<0$ 05 were defined as statistically significant.

\section{Results}

Participant characteristics were shown in Table 1. Compare to control, cases reported higher smoker and lower literate patients. Also, there were significant differences between both groups in terms of BMI, FBS, $\mathrm{BS} 2 \mathrm{Hp}$, and A1C. Cases and controls were not significantly different in terms of mean age, BS2Hp, job, marriage, gender, physical activity, and economic status. Significant differences were found in terms of age and smokers across the quartiles of the DII score. The second quartile of DII was associated with younger age and higher smokers. There were no other significant differences across categories of the DII. 
Table 1

General characteristics of study participants*

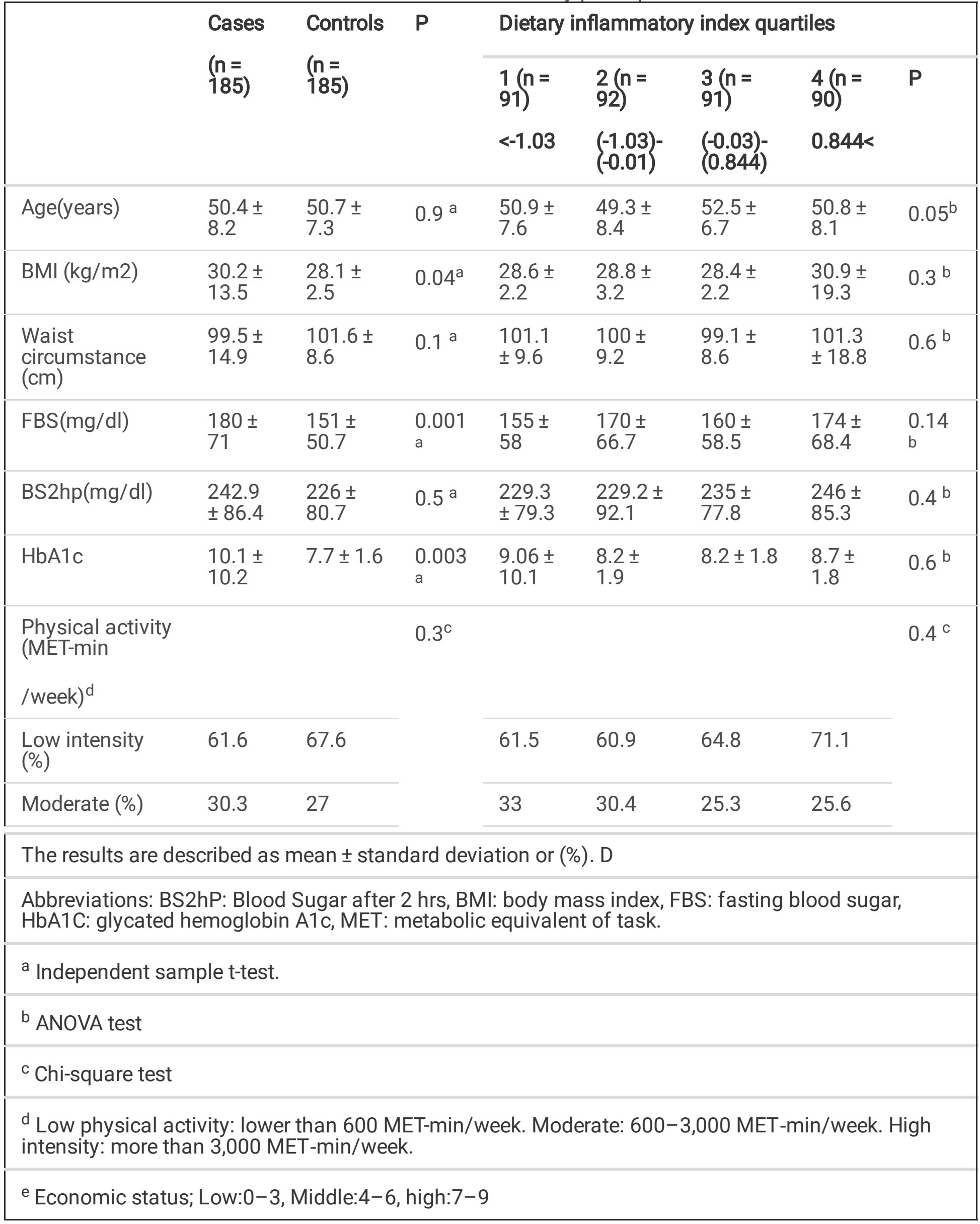




\begin{tabular}{|c|c|c|c|c|c|c|c|c|}
\hline & Cases & Controls & $P$ & Dietary & flammato & index qu & tiles & \\
\hline & $\begin{array}{l}(n= \\
185)\end{array}$ & $\begin{array}{l}(n= \\
185)\end{array}$ & & $\begin{array}{l}1(n= \\
91)^{n}\end{array}$ & $\begin{array}{l}2(n= \\
92)\end{array}$ & $\begin{array}{l}3(n= \\
91)\end{array}$ & $\begin{array}{l}4(n= \\
90)\end{array}$ & $P$ \\
\hline & & & & $<-1.03$ & $\left(\begin{array}{l}-1.03) \\
(-0.01)\end{array}\right.$ & $\begin{array}{l}(-0.03)- \\
(0.844)\end{array}$ & $0.844<$ & \\
\hline $\begin{array}{l}\text { High intensity } \\
\text { (\%) }\end{array}$ & 8.1 & 5.4 & & 5.5 & 8.7 & 9.9 & 3.3 & \\
\hline Females (\%) & 63.8 & 63.8 & $0.5^{c}$ & 64.8 & 58.7 & 65.9 & 58 & $0.7^{c}$ \\
\hline Married (\%) & 99.5 & 100 & $\begin{array}{l}0.66 \\
c\end{array}$ & 25 & 25.5 & 27 & 23 & $\begin{array}{l}< \\
0.001 \\
c\end{array}$ \\
\hline Education (\%) & & & $\begin{array}{l}< \\
0.001 \\
c\end{array}$ & 32 & 16 & 18 & 34 & $\begin{array}{l}0.08 \\
c\end{array}$ \\
\hline Literate (\%) & 81.1 & 94.6 & & 90.1 & 90.2 & 86.8 & 83.3 & $0.4^{c}$ \\
\hline Illiterate (\%) & 18.9 & 5.4 & & 9.9 & 9.8 & 13.2 & 16.7 & \\
\hline $\begin{array}{l}\text { Current smoker } \\
(\%)\end{array}$ & 10.3 & 4.3 & $\begin{array}{l}0.02 \\
c\end{array}$ & 4.4 & 13 & 3.3 & 8.9 & $\begin{array}{l}0.04 \\
c\end{array}$ \\
\hline $\begin{array}{l}\text { Economic status } \\
\mathrm{e}\end{array}$ & & & $0.5^{c}$ & & & & & \\
\hline Low (\%) & 15.1 & 17.3 & & 15.4 & 19.6 & 14.3 & 16.7 & $0.5^{c}$ \\
\hline Middle (\%) & 83.3 & 79.5 & & 82.4 & 79.3 & 81.3 & 81.1 & \\
\hline High (\%) & 1.6 & 3.2 & & 2.2 & 1.1 & 4.4 & 2.2 & \\
\hline Job & & & $0.2^{c}$ & & & & & $0.2^{c}$ \\
\hline The results are de & cribed as & hean \pm stan & dard dev & tion or ( & & & & \\
\hline $\begin{array}{l}\text { Abbreviations: BS } \\
\text { HbA1C: glycated l }\end{array}$ & $\begin{array}{l}\text { hP: Bloo } \\
\text { emoglobi }\end{array}$ & $\begin{array}{l}\text { Sugar after } \\
\text { A1c, MET: }\end{array}$ & $\begin{array}{l}2 \mathrm{hrs}, \mathrm{BN} \\
\text { רetaboli }\end{array}$ & $\begin{array}{l}\text { : body } \\
\text { equival }\end{array}$ & $\begin{array}{l}\text { ss index, } \mathrm{F} \\
\text { of task. }\end{array}$ & S: fasting & ood sug & \\
\hline${ }^{\mathrm{a}}$ Independent sar & ple t-test. & & & & & & & \\
\hline${ }^{\mathrm{b}}$ ANOVA test & & & & & & & & \\
\hline${ }^{\mathrm{C}}$ Chi-square test & & & & & & & & \\
\hline $\begin{array}{l}{ }^{d} \text { Low physical ac } \\
\text { intensity: more th }\end{array}$ & $\begin{array}{l}\text { ity: lowe } \\
3,000 \mathrm{~N}\end{array}$ & $\begin{array}{l}\text { an } 600 \mathrm{M} \\
-\mathrm{min} / \mathrm{wee}\end{array}$ & T-min/ & ek. Mo & ate: 600 &, $000 \mathrm{MET}$ & n/week. & ligh \\
\hline
\end{tabular}




\begin{tabular}{|c|c|c|c|c|c|c|c|c|}
\hline \multirow{3}{*}{ 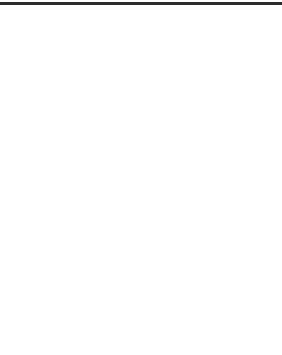 } & \multirow{3}{*}{$\begin{array}{l}\text { Cases } \\
(n= \\
185)\end{array}$} & \multirow{3}{*}{$\begin{array}{l}\text { Controls } \\
(n= \\
185)\end{array}$} & \multirow[t]{3}{*}{$\mathbf{P}$} & \multicolumn{5}{|c|}{ Dietary inflammatory index quartiles } \\
\hline & & & & \multirow{2}{*}{$\begin{array}{l}1(n= \\
91)^{n} \\
<-1.03\end{array}$} & \multirow{2}{*}{$\begin{array}{l}2(n= \\
92) \\
(-1.03)- \\
(-0.01)\end{array}$} & \multirow{2}{*}{$\begin{array}{l}3(n= \\
91) \\
(-0.03)- \\
(0.844)\end{array}$} & $\begin{array}{l}4(n= \\
90)\end{array}$ & \multirow[t]{2}{*}{$\mathbf{P}$} \\
\hline & & & & & & & $0.844<$ & \\
\hline Working (\%) & 43.8 & 48.6 & & 42.9 & 55.4 & 45.1 & 43.3 & \\
\hline Housewife & 56.2 & 51.4 & & 57.1 & 44.6 & 54.9 & 56.7 & \\
\hline \multicolumn{9}{|c|}{ The results are described as mean \pm standard deviation or (\%). D } \\
\hline \multicolumn{9}{|c|}{$\begin{array}{l}\text { Abbreviations: BS2hP: Blood Sugar after } 2 \text { hrs, BMl: body mass index, FBS: fasting blood sugar, } \\
\text { HbA1C: glycated hemoglobin A1c, MET: metabolic equivalent of task. }\end{array}$} \\
\hline \multicolumn{9}{|c|}{ a Independent sample t-test. } \\
\hline \multicolumn{9}{|l|}{${ }^{\mathrm{b}}$ ANOVA test } \\
\hline \multicolumn{9}{|c|}{${ }^{\mathrm{c}}$ Chi-square test } \\
\hline \multicolumn{9}{|c|}{$\begin{array}{l}\text { d Low physical activity: lower than } 600 \text { MET-min/week. Moderate: } 600-3,000 \text { MET-min/week. High } \\
\text { intensity: more than 3,000 MET-min/week. }\end{array}$} \\
\hline \multicolumn{9}{|c|}{${ }^{\mathrm{e}}$ Economic status; Low:0-3, Middle:4-6, high:7-9 } \\
\hline
\end{tabular}

Table 2 presents the dietary intake of participants. Cases had a higher intake of energy, PUFA, MUFA, Mg, dietary fiber, refined grains, red and processed meat, Green/black tea, and coffee compared to controls. However, lower consumption of vitamin E, whole grains, white meat, fish, fruits, vegetables, the dairy products was found in cases. The first quartile of DII was associated with higher intake of energy $(\mathrm{p}<$ $0.001)$, PUFA $(p<0.001), F e(p<0.001), M g(p<0.001), Z n(p=0.001)$, vitamin $A(p<0.001)$, vitamin $C(p=$ $0.03)$, vitamin $E(p<0.001)$, b-carotene $(p<0.001)$, fish $(p<0.001)$, vegetables $(p<0.001)$, legume and nuts $(p=0.001)$, Green/black tea and coffee $(p<0.001)$. Also, there were significant differences in terms of fruits $(p<0.001)$, dietary fiber $(p<0.001)$, vitamin $D(p=0.02)$, folate $(p<0.001)$, niacin $(p=0.008)$, thiamin $(p$ $<0.001)$, vitamin B12 $(p=0.04)$, SFA $(p<0.001)$, total fat $(p=0.002)$, carbohydrate $(p<0.001)$ across the quartile of the DII score. 
Table 2

Dietary intakes of patients

\begin{tabular}{|c|c|c|c|c|c|c|c|c|}
\hline & \multirow{3}{*}{$\begin{array}{l}\text { Cases } \\
(n= \\
185)\end{array}$} & \multirow{3}{*}{$\begin{array}{l}\text { Controls } \\
(n=185)\end{array}$} & \multirow[t]{3}{*}{$P *$} & \multicolumn{4}{|c|}{ Dietary inflammatory index quartiles } & \multirow[t]{3}{*}{$P^{*}$} \\
\hline & & & & $\begin{array}{l}1(n= \\
91)\end{array}$ & $\begin{array}{l}2(n= \\
92)\end{array}$ & $\begin{array}{l}3(n= \\
91)\end{array}$ & $\begin{array}{l}4(n= \\
90)\end{array}$ & \\
\hline & & & & $<-1.03$ & $\left(\begin{array}{c}-1.03) \\
(-0.01)\end{array}\right)$ & $\left(\begin{array}{l}-0.03)- \\
(0.844)\end{array}\right.$ & $0.844<$ & \\
\hline & $\begin{array}{l}\text { Mean } \\
\pm S E\end{array}$ & $\begin{array}{l}\text { Mean } \pm \\
\text { SE }\end{array}$ & & $\begin{array}{l}\text { Mean } \\
\pm \text { SE }\end{array}$ & $\begin{array}{l}\text { Mean } \\
\pm \text { SE }\end{array}$ & $\begin{array}{l}\text { Mean } \\
\pm S E\end{array}$ & $\begin{array}{l}\text { Mean } \\
\pm S E\end{array}$ & \\
\hline Energy $(\mathrm{kcal} / \mathrm{d})$ & $\begin{array}{l}2724 \\
\pm 31.3\end{array}$ & $\begin{array}{l}2519 \pm \\
31.3\end{array}$ & $<.001$ & $\begin{array}{l}2834 \\
\pm 41.5\end{array}$ & $\begin{array}{l}2755 \\
\pm 41.6\end{array}$ & $\begin{array}{l}2501 \pm \\
41.8\end{array}$ & $\begin{array}{l}2385 \\
\pm 41.8\end{array}$ & $\begin{array}{l}<.001 \\
0.00\end{array}$ \\
\hline \multicolumn{9}{|l|}{ Nutrients } \\
\hline $\begin{array}{l}\text { Carbohydrates } \\
(\mathrm{g} / \mathrm{d})\end{array}$ & $\begin{array}{l}393 \pm \\
2.9\end{array}$ & $399 \pm 2.9$ & 0.15 & $\begin{array}{l}401.3 \\
\pm 3.56\end{array}$ & $\begin{array}{l}404.6 \\
\pm 3.4\end{array}$ & $\begin{array}{l}400.5 \\
\pm 3.4\end{array}$ & $\begin{array}{l}383.9 \\
\pm 3.6\end{array}$ & $\hat{0}_{0.001}$ \\
\hline Proteins $(\mathrm{g} / \mathrm{d})$ & $\begin{array}{l}117 \pm \\
15.4\end{array}$ & $\begin{array}{l}120 \pm \\
15.4\end{array}$ & 0.9 & $\begin{array}{l}103.8 \\
\pm 2.8\end{array}$ & $\begin{array}{l}101.5 \\
\pm 2.8\end{array}$ & $\begin{array}{l}106 \pm \\
2.8\end{array}$ & $\begin{array}{l}106 \pm \\
2.8\end{array}$ & 0.6 \\
\hline Total fats (g/d) & $\begin{array}{l}81.3 \\
\pm 2.2\end{array}$ & $\begin{array}{l}74.6 \pm \\
2.2\end{array}$ & 0.03 & $\begin{array}{l}74.08 \\
\pm 1.3\end{array}$ & $\begin{array}{l}74.3 \pm \\
1.2\end{array}$ & $\begin{array}{l}75 \pm \\
1.2\end{array}$ & $\begin{array}{l}80.4 \pm \\
1.3\end{array}$ & 0.002 \\
\hline $\begin{array}{l}\text { Cholesterol } \\
\text { (mg/d) }\end{array}$ & $\begin{array}{l}232 \pm \\
4.2\end{array}$ & $229 \pm 4.2$ & 0.6 & $\begin{array}{l}221 \pm \\
6.1\end{array}$ & $\begin{array}{l}233 \pm \\
5.9\end{array}$ & $\begin{array}{l}237 \pm \\
5.9\end{array}$ & $\begin{array}{l}235 \pm \\
6.1\end{array}$ & 0.2 \\
\hline $\operatorname{SFA}(g / d)$ & $\begin{array}{l}24.3 \\
\pm 1.2\end{array}$ & $\begin{array}{l}21.9 \pm \\
1.2\end{array}$ & 0.16 & $\begin{array}{l}19.9 \\
\pm 0.39\end{array}$ & $\begin{array}{l}20.9 \pm \\
0.38\end{array}$ & $\begin{array}{l}21.8 \pm \\
0.38\end{array}$ & $\begin{array}{l}25.1 \pm \\
0.39\end{array}$ & $\begin{array}{l}<.001 \\
0.00\end{array}$ \\
\hline MUFA (g/d) & $\begin{array}{l}25.8 \\
\pm 0.2\end{array}$ & $\begin{array}{l}24.5 \pm \\
0.2\end{array}$ & $\begin{array}{l}< \\
0.001\end{array}$ & $\begin{array}{l}25.2 \\
\pm 0.36\end{array}$ & $\begin{array}{l}24.9 \pm \\
0.35\end{array}$ & $\begin{array}{l}24.8 \pm \\
0.35\end{array}$ & $\begin{array}{l}25.8 \pm \\
0.36\end{array}$ & 0.2 \\
\hline PUFA (g/d) & $\begin{array}{l}18.2 \\
\pm 0.1\end{array}$ & $\begin{array}{l}17.3 \pm \\
0.1\end{array}$ & $\begin{array}{l}< \\
0.001\end{array}$ & $\begin{array}{l}18.8 \\
\pm 0.25\end{array}$ & $\begin{array}{l}18 \pm \\
0.24\end{array}$ & $\begin{array}{l}17.2 \pm \\
0.24\end{array}$ & $\begin{array}{l}16.8 \pm \\
0.25\end{array}$ & $\begin{array}{l}<.001 \\
0.00\end{array}$ \\
\hline $\mathrm{Fe}(\mathrm{mg} / \mathrm{d})$ & $\begin{array}{l}33.1 \\
\pm 7.6\end{array}$ & $\begin{array}{l}31.4 \pm \\
7.6\end{array}$ & 0.8 & $\begin{array}{l}25.7 \\
\pm 0.2\end{array}$ & $\begin{array}{l}25.3 \pm \\
0.27\end{array}$ & $\begin{array}{l}24.7 \pm \\
0.27\end{array}$ & $\begin{array}{l}23.5 \pm \\
0.28\end{array}$ & $\begin{array}{l}<.001 \\
0.001\end{array}$ \\
\hline $\mathrm{Mg}(\mathrm{mg} / \mathrm{d})$ & $\begin{array}{l}505 \pm \\
7.3\end{array}$ & $597 \pm 7.3$ & $<.001$ & $\begin{array}{l}597 \pm \\
183\end{array}$ & $\begin{array}{l}530 \pm \\
141\end{array}$ & $\begin{array}{l}484 \pm \\
107\end{array}$ & $\begin{array}{l}483 \pm \\
125\end{array}$ & <. 001 \\
\hline $\mathrm{Zn}(\mathrm{mg} / \mathrm{d})$ & $\begin{array}{l}17.5 \\
\pm 3.2\end{array}$ & $\begin{array}{l}17.7 \pm \\
3.2\end{array}$ & 0.9 & $\begin{array}{l}15.2 \\
\pm 0.23\end{array}$ & $\begin{array}{l}14.1 \pm \\
0.22\end{array}$ & $\begin{array}{l}14.6 \pm \\
0.22\end{array}$ & $\begin{array}{l}13.9 \pm \\
0.23\end{array}$ & 0.001 \\
\hline $\mathrm{Se}(\mathrm{mg} / \mathrm{d})$ & $\begin{array}{l}194 \pm \\
14.2\end{array}$ & $\begin{array}{l}208 \pm \\
14.1\end{array}$ & 0.5 & $\begin{array}{l}189.6 \\
\pm 20.6\end{array}$ & $\begin{array}{l}183.3 \\
\pm 20.2\end{array}$ & $\begin{array}{l}188.1 \\
\pm 20.2\end{array}$ & $\begin{array}{l}250 \pm \\
20.8\end{array}$ & 0.07 \\
\hline $\begin{array}{l}\text { Thiamine } \\
\text { (mg/d) }\end{array}$ & $\begin{array}{l}2.9 \pm \\
0.02\end{array}$ & $3 \pm 0.02$ & 0.43 & $\begin{array}{l}2.9 \pm \\
0.02\end{array}$ & $\begin{array}{l}3.05 \pm \\
0.02\end{array}$ & $\begin{array}{l}2.9 \pm \\
0.02\end{array}$ & $\begin{array}{l}2.8 \pm \\
0.02\end{array}$ & $\dot{0} 001$ \\
\hline
\end{tabular}

*All values were adjusted for age, sex and energy, except for dietary energy intake, which was only adjusted for age and sex using ANCOVA. 


\begin{tabular}{|c|c|c|c|c|c|c|c|c|}
\hline & \multirow{3}{*}{$\begin{array}{l}\text { Cases } \\
(n= \\
185)\end{array}$} & \multirow{3}{*}{$\begin{array}{l}\text { Controls } \\
(n=185)\end{array}$} & \multirow[t]{3}{*}{$P^{*}$} & \multicolumn{4}{|c|}{ Dietary inflammatory index quartiles } & \multirow[t]{3}{*}{$P *$} \\
\hline & & & & $\begin{array}{l}1(n= \\
91)\end{array}$ & $\begin{array}{l}2(n= \\
92)\end{array}$ & $\begin{array}{l}3(n= \\
91)\end{array}$ & $\begin{array}{l}4(n= \\
90)\end{array}$ & \\
\hline & & & & $<-1.03$ & $\left(\begin{array}{l}-1.03) \\
(-0.01)\end{array}\right.$ & $\begin{array}{l}(-0.03)- \\
(0.844)\end{array}$ & $0.844<$ & \\
\hline $\begin{array}{l}\text { Riboflavin } \\
\text { (mg/d) }\end{array}$ & $\begin{array}{l}2.5 \pm \\
0.14\end{array}$ & $\begin{array}{l}2.6 \pm \\
0.14\end{array}$ & 0.5 & $\begin{array}{l}2.5 \pm \\
0.2\end{array}$ & $\begin{array}{l}2.4 \pm \\
0.2\end{array}$ & $\begin{array}{l}2.40 \pm \\
0.2\end{array}$ & $\begin{array}{l}3.04 \pm \\
0.2\end{array}$ & 0.1 \\
\hline $\operatorname{Niacin}(\mathrm{mg} / \mathrm{d})$ & $\begin{array}{l}31.2 \\
\pm 0.48\end{array}$ & $\begin{array}{l}32.6 \pm \\
0.48\end{array}$ & 0.05 & $\begin{array}{l}32.2 \\
\pm 0.66\end{array}$ & $\begin{array}{l}32.5 \pm \\
0.65\end{array}$ & $\begin{array}{l}33.3 \pm \\
0.65\end{array}$ & $\begin{array}{l}30.2 \pm \\
0.67\end{array}$ & 0.008 \\
\hline $\begin{array}{l}\text { Vitamin B6 } \\
(\mathrm{mg} / \mathrm{d})\end{array}$ & $\begin{array}{l}5.5 \pm \\
3.2\end{array}$ & $5.3 \pm 3.2$ & 0.9 & $\begin{array}{l}1.4 \pm \\
4.7\end{array}$ & $\begin{array}{l}1.1 \pm \\
4.6\end{array}$ & $\begin{array}{l}2.3 \pm \\
4.6\end{array}$ & $\begin{array}{l}15.7 \pm \\
4.7\end{array}$ & 0.1 \\
\hline $\begin{array}{l}\text { b-carotene } \\
\text { (mcg/d) }\end{array}$ & $\begin{array}{l}3028 \\
\pm 188\end{array}$ & $\begin{array}{l}3502 \pm \\
188\end{array}$ & 0.08 & $\begin{array}{l}4911 \\
\pm 259\end{array}$ & $\begin{array}{l}2980 \\
\pm 253\end{array}$ & $\begin{array}{l}2814 \pm \\
254\end{array}$ & $\begin{array}{l}2414 \\
\pm 262\end{array}$ & $\begin{array}{l}< \\
0.001\end{array}$ \\
\hline $\begin{array}{l}\text { Vitamin } A \\
(R E / d)\end{array}$ & $\begin{array}{l}619.9 \\
\pm 32.3\end{array}$ & $\begin{array}{l}605.6 \pm \\
32.8\end{array}$ & 0.7 & $\begin{array}{l}736 \pm \\
36.7\end{array}$ & $\begin{array}{l}533 \pm \\
35.9\end{array}$ & $\begin{array}{l}523 \pm \\
36\end{array}$ & $\begin{array}{l}583 \pm \\
37.1\end{array}$ & $\begin{array}{l}<.001 \\
0.00\end{array}$ \\
\hline $\begin{array}{l}\text { Vitamin } C \\
(\mathrm{mg} / \mathrm{d})\end{array}$ & $\begin{array}{l}91 \pm \\
20.8\end{array}$ & $\begin{array}{l}144 \pm \\
20.8\end{array}$ & 0.07 & $\begin{array}{l}125 \pm \\
10.6\end{array}$ & $\begin{array}{l}95.5 \pm \\
10.4\end{array}$ & $\begin{array}{l}84.9 \pm \\
10.4\end{array}$ & $\begin{array}{l}89.3 \pm \\
10.8\end{array}$ & 0.03 \\
\hline $\begin{array}{l}\text { Vitamin } E \\
(\mathrm{mg} / \mathrm{d})\end{array}$ & $\begin{array}{l}12.8 \\
\pm 0.21\end{array}$ & $\begin{array}{l}14.1 \pm \\
0.21\end{array}$ & $<0.001$ & $\begin{array}{l}16 \pm \\
0.25\end{array}$ & $\begin{array}{l}14.1 \pm \\
0.24\end{array}$ & $\begin{array}{l}13 \pm \\
0.24\end{array}$ & $\begin{array}{l}11 \pm \\
0.25\end{array}$ & $\begin{array}{l}<.001 \\
0.001\end{array}$ \\
\hline $\begin{array}{l}\text { Vitamin D } \\
(\mathrm{mcg} / \mathrm{d})\end{array}$ & $\begin{array}{l}1.9 \pm \\
0.17\end{array}$ & $\begin{array}{l}2.1 \pm \\
0.17\end{array}$ & 0.3 & $\begin{array}{l}1.9 \pm \\
0.13\end{array}$ & $\begin{array}{l}1.5 \pm \\
0.13\end{array}$ & $\begin{array}{l}2 \pm \\
0.13\end{array}$ & $\begin{array}{l}2.1 \pm \\
0.13\end{array}$ & 0.02 \\
\hline $\begin{array}{l}\text { Vitamin B12 } \\
(\mathrm{mcg} / \mathrm{d})\end{array}$ & $\begin{array}{l}10.5 \\
\pm 5.4\end{array}$ & $8.7 \pm 5.4$ & 0.8 & $\begin{array}{l}4.2 \pm \\
0.35\end{array}$ & $\begin{array}{l}3.9 \pm \\
0.34\end{array}$ & $\begin{array}{l}3.9 \pm \\
0.34\end{array}$ & $\begin{array}{l}5.1 \pm \\
0.35\end{array}$ & 0.04 \\
\hline Folate (mcg/d) & $\begin{array}{l}743 \pm \\
7.3\end{array}$ & $737 \pm 7.3$ & 0.5 & $\begin{array}{l}767 \pm \\
8.2\end{array}$ & $\begin{array}{l}774 \pm \\
8.1\end{array}$ & $\begin{array}{l}741 \pm \\
8.1\end{array}$ & $\begin{array}{l}692 \pm \\
8.3\end{array}$ & $\begin{array}{l}< \\
0.001\end{array}$ \\
\hline $\begin{array}{l}\text { Dietary fiber } \\
(\mathrm{g} / \mathrm{d})\end{array}$ & $\begin{array}{l}79.8 \\
\pm 1.4\end{array}$ & $\begin{array}{l}71.7 \pm \\
1.4\end{array}$ & $<0.001$ & $\begin{array}{l}74.9 \\
\pm 1.9\end{array}$ & $\begin{array}{l}83.3 \pm \\
1.9\end{array}$ & $\begin{array}{l}75.3 \pm \\
1.9\end{array}$ & $\begin{array}{l}70.9 \pm \\
1.9\end{array}$ & $\begin{array}{l}< \\
0.001\end{array}$ \\
\hline \multicolumn{9}{|l|}{ Food groups } \\
\hline $\begin{array}{l}\text { Refined grains } \\
(\mathrm{g} / \mathrm{d})\end{array}$ & $\begin{array}{l}464 \pm \\
9.6\end{array}$ & $369 \pm 9.6$ & $<001$ & $\begin{array}{l}413 \pm \\
14.9\end{array}$ & $\begin{array}{l}436 \pm \\
14.6\end{array}$ & $\begin{array}{l}410 \pm \\
14.7\end{array}$ & $\begin{array}{l}401 \pm \\
15.1\end{array}$ & 0.4 \\
\hline $\begin{array}{l}\text { Whole-grains } \\
\text { (g/d }\end{array}$ & $\begin{array}{l}219 \pm \\
8.5\end{array}$ & $291 \pm 8.5$ & $\begin{array}{l}< \\
0.001\end{array}$ & $\begin{array}{l}254 \pm \\
13\end{array}$ & $\begin{array}{l}234 \pm \\
12.7\end{array}$ & $\begin{array}{l}261 \pm \\
12.7\end{array}$ & $\begin{array}{l}270 \pm \\
13.1\end{array}$ & 0.2 \\
\hline $\begin{array}{l}\text { White meats } \\
(\mathrm{g} / \mathrm{d})\end{array}$ & $\begin{array}{l}49.8 \pm \\
3.3\end{array}$ & $\begin{array}{l}40.4 \pm \\
3.3\end{array}$ & 0.05 & $\begin{array}{l}41.9 \\
\pm 4.9\end{array}$ & $\begin{array}{l}43.7 \pm \\
4.8\end{array}$ & $\begin{array}{l}55.4 \pm \\
4.8\end{array}$ & $\begin{array}{l}39.2 \pm \\
4.9\end{array}$ & 0.08 \\
\hline $\begin{array}{l}\text { Red and } \\
\text { processed } \\
\text { meats }(\mathrm{g} / \mathrm{d})\end{array}$ & $\begin{array}{l}7.1 \pm \\
0.2\end{array}$ & $5.2 \pm 0.2$ & $\begin{array}{l}< \\
0.001\end{array}$ & $\begin{array}{l}5.9 \pm \\
0.38\end{array}$ & $\begin{array}{l}6.2 \pm \\
0.38\end{array}$ & $\begin{array}{l}6.3 \pm \\
0.38\end{array}$ & $\begin{array}{l}6 \pm \\
0.39\end{array}$ & 0.9 \\
\hline
\end{tabular}




\begin{tabular}{|c|c|c|c|c|c|c|c|c|}
\hline & \multirow{3}{*}{$\begin{array}{l}\text { Cases } \\
(n= \\
185)\end{array}$} & \multirow{3}{*}{$\begin{array}{l}\text { Controls } \\
(n=185)\end{array}$} & \multirow[t]{3}{*}{$P^{*}$} & \multicolumn{4}{|c|}{ Dietary inflammatory index quartiles } & \multirow[t]{3}{*}{ P* } \\
\hline & & & & $\begin{array}{l}1(n= \\
91)\end{array}$ & $\begin{array}{l}2(n= \\
92)\end{array}$ & $\begin{array}{l}3(n= \\
91)\end{array}$ & $\begin{array}{l}4(n= \\
90)\end{array}$ & \\
\hline & & & & $<-1.03$ & $\left(\begin{array}{l}-1.03)- \\
-0.01)\end{array}\right.$ & $\begin{array}{l}(-0.03)- \\
(0.844)\end{array}$ & $0.844<$ & \\
\hline Fish $(g / d)$ & $\begin{array}{l}2.4 \pm \\
0.1\end{array}$ & $4 \pm 0.1$ & $\begin{array}{l}<.001 \\
0 .\end{array}$ & $\begin{array}{l}4.3 \pm \\
0.2\end{array}$ & $\begin{array}{l}3.4 \pm \\
0.2\end{array}$ & $\begin{array}{l}3.2 \pm \\
0.2\end{array}$ & $\begin{array}{l}1.7 \pm \\
0.2\end{array}$ & $\begin{array}{l}< \\
0.001\end{array}$ \\
\hline Fruits $(\mathrm{g} / \mathrm{d})$ & $\begin{array}{l}137 \pm \\
2.9\end{array}$ & $216 \pm 2.9$ & $<.001$ & $\begin{array}{l}185 \pm \\
5.8\end{array}$ & $\begin{array}{l}193 \pm \\
5.6\end{array}$ & $\begin{array}{l}180.4 \\
\pm 5.6\end{array}$ & $\begin{array}{l}148 \pm \\
5.8\end{array}$ & <. 001 \\
\hline $\begin{array}{l}\text { Vegetables } \\
(\mathrm{g} / \mathrm{d})\end{array}$ & $\begin{array}{l}295 \pm \\
7.5\end{array}$ & $427 \pm 7.5$ & $\begin{array}{l}< \\
0.001\end{array}$ & $\begin{array}{l}454 \pm \\
11.1\end{array}$ & $\begin{array}{l}365 \pm \\
11.1\end{array}$ & $\begin{array}{l}353 \pm \\
11.1\end{array}$ & $\begin{array}{l}276 \pm \\
11.4\end{array}$ & $\begin{array}{l}<.001 \\
0.001\end{array}$ \\
\hline $\begin{array}{l}\text { Dairy products } \\
(\mathrm{g} / \mathrm{d})\end{array}$ & $\begin{array}{l}308 \pm \\
8\end{array}$ & $400 \pm 8$ & $\begin{array}{l}<.001 \\
0.001\end{array}$ & $\begin{array}{l}360 \pm \\
12.5\end{array}$ & $\begin{array}{l}336 \pm \\
12.4\end{array}$ & $\begin{array}{l}372 \pm \\
12.3\end{array}$ & $\begin{array}{l}340 \pm \\
12.8\end{array}$ & 0.1 \\
\hline $\begin{array}{l}\text { Legumes and } \\
\text { nuts }(\mathrm{g} / \mathrm{d})\end{array}$ & $\begin{array}{l}77.4 \\
\pm 1.5\end{array}$ & $\begin{array}{l}78.1 \pm \\
1.5\end{array}$ & 0.7 & $\begin{array}{l}80.9 \\
\pm 2.1\end{array}$ & $\begin{array}{l}79.2 \pm \\
2.1\end{array}$ & $\begin{array}{l}79 \pm \\
2.1\end{array}$ & $\begin{array}{l}69.5 \pm \\
2.2\end{array}$ & 0.001 \\
\hline $\begin{array}{l}\text { Green/black tea } \\
\text { and coffee }(\mathrm{g} / \mathrm{d})\end{array}$ & $\begin{array}{l}635 \pm \\
15.6\end{array}$ & $\begin{array}{l}568 \pm \\
15.6\end{array}$ & 0.003 & $\begin{array}{l}718 \pm \\
21.3\end{array}$ & $\begin{array}{l}654 \pm \\
20.9\end{array}$ & $\begin{array}{l}551 \pm \\
20.9\end{array}$ & $\begin{array}{l}492 \pm \\
21.6\end{array}$ & $<.001$ \\
\hline
\end{tabular}

Table 3 showed the Multivariable-adjusted ORs and 95\% Cls for the association of DII score and odd of DSPN. There was a significant trend across the quartiles of DII for the crude model ( $p$-trend- 0.009) (OR = $2.6,95 \% \mathrm{Cl}=1.4-4.8)$, after adjustment for age, sex, energy in model 1 ( $p$-trend $<0.001)(\mathrm{OR}=0.25,95 \%$ $\mathrm{Cl}=0.13-0.49$ ), additionally adjusted for physical activity, education, smokers, FBS, A1C and economic status in model $2(\mathrm{OR}=0.27,95 \% \mathrm{Cl}=0.13-0.57)$ ( $p$-trend $<0.001)$. The association was not altered substantially by further adjustment for $\mathrm{BMI}$ in model $3(\mathrm{OR}=6.3,95 \% \mathrm{Cl}=2.7-14.6)(\mathrm{p}$-trend $<0.001)$. 
Table 3

Odds ratios (ORs) and $95 \%$ confidence intervals (95\% Cls) of DSPN according to quartiles of dietary inflammatory index

\begin{tabular}{|c|c|c|c|c|c|}
\hline & Dietary inf & nmatory index qu & & & P-trend* \\
\hline & $1(n=91)$ & $2(n=92)$ & $3(n=91)$ & $4(n=90)$ & \\
\hline & $<-1.03$ & $(-1.03)-(-0.01)$ & $(-0.03)-(0.844)$ & $0.844<$ & \\
\hline & OR & OR $(95 \% \mathrm{Cl})$ & OR $(95 \% \mathrm{Cl})$ & OR $(95 \% \mathrm{Cl})$ & \\
\hline Crude & 1.00 & $1.3(0.77-2.5)$ & $0.95(0.53-1.7)$ & $2.6(1.4-4.8)$ & 0.009 \\
\hline Model 1 & 1.00 & $0.14(0.07-0.3)$ & $0.25(0.12-0.5)$ & $0.25(0.13-0.49)$ & 0.0001 \\
\hline Model 2 & 1.00 & $0.16(0.07-0.3)$ & $0.26(0.12-0.56)$ & $0.27(0.13-0.57)$ & $<0.001$ \\
\hline Model 3 & 1.00 & $1.5(0.7-3.2)$ & $1.7(0.8-3.7)$ & $6.3(2.7-14.6)$ & $<0.001$ \\
\hline $\begin{array}{l}\text { * Binary I } \\
\text { increasin } \\
\text { variable }\end{array}$ & $\begin{array}{l}\text { tic regres } \\
\text { artile was }\end{array}$ & $\begin{array}{l}\text { was used to ol } \\
\text { mined by cons }\end{array}$ & $\begin{array}{l}\text { R and } 95 \% \mathrm{Cl} .7 \\
\mathrm{~g} \text { the median sc }\end{array}$ & $\begin{array}{l}\text { erall trend of OR } \\
\text { each category as }\end{array}$ & intinuous \\
\hline Model 1: & djusted for a & (continuous), sex & ale/female) and er & intake $(\mathrm{kJ} / \mathrm{d}$ or $\mathrm{kc}$ & \\
\hline $\begin{array}{l}\text { Model 2: } \\
\text { educatio } \\
\text { (continuc }\end{array}$ & $\begin{array}{l}\text { ther adjust } \\
\text { terate, illite } \\
\text {, } \mathrm{HbA} 1 \mathrm{C}(\mathrm{c}\end{array}$ & $\begin{array}{l}\text { nts were made fo } \\
\text { e), smoking statc } \\
\text { tinuous), BS2Hp }\end{array}$ & $\begin{array}{l}\text { hysical activity (low } \\
\text { es, no), economic } \\
\text { tinuous). }\end{array}$ & $\begin{array}{l}\text { ensity, moderate, hi } \\
\text { us (low, middle, hig }\end{array}$ & $\begin{array}{l}\text { intensity), } \\
=\text { BS }\end{array}$ \\
\hline Model 3: & ditionally a & sted for BMI (co & ous). & & \\
\hline
\end{tabular}

\section{Discussion}

To the best of our knowledge, no study has yet explored the relationship between DII score and risk of DSPN. Our results indicated a positive relationship between the DII score and the odds of DSPN. This study provides some evidence to support the recommendation of low consumption of pro-inflammatory foods and a high intake of foods with anti-inflammatory potential. The association was not changed after adjustment for BMI, age, sex, and other risk factors.

In line with our findings, the results of a case-control study conducted on women showed that a diet that was associated with inflammatory markers can result in inflammation and increasing the developing of type 2 diabetes[13]. A cross-sectional study investigated the relation between DII and T2DM in a Mexican population. The results showed that patients with higher DII score had about three times greater odds of diabetes compared to subjects with lower DII score[14]. A case-control study investigating the association between DII and pre-diabetes indicated that patients with higher DII score was at increased odds of prediabetes compared to subjects who had a more anti-inflammatory diet [15]. Although a trial study showed that a diet rich in vegetables, fruits, grains, and legumes improved diabetic neuropathy pain [22], we have found no other study that used the DII. 
In the animal study, inflammation has been considered as one of the main important risk factors in the pathogenies of DSPN[23,24]. Some studies have shown the connection between diet and chronic inflammation $[12,25,26]$. The Inflammatory potential of a diet can play an important role in the development and pathogenies of diseases including T2DM[14], cancers [27].

The mechanisms between the anti-inflammatory diet and DSPN could be explained by improving hyperglycemia that has been related to the inflammatory process. Chronic hyperglycemia results in the activation of several pathways including the elevation of protein kinase $\mathrm{C}$, polyol pathway, glucose autoxidation, hexosamine flux. Therefore, hyperglycemia through theses pathways activate proinflammatory cytokines such as interleukin-1, interleukin- 6 and finally nuclear factor kappa-light-chainenhancer of activated B cells (NF-KB) which play a central role in inflammation[28-30].

There are some strengths to this study. First, it was adjusted for various covariate that could change the result. Second, new cases of DSPN were enrolled in order to reduce the bias from changed dietary habits in participants. Third, this is the first study that investigated the association between DII and DSPN.

There are some limitations to this study. First, since this is a case-control study, cause and effect results cannot be concluded. Second, the FFQ was used for assessing food intake which carried recall bias and misclassification remains a possibility. Third, since we used FFQ for calculation of DII, some parameters of the DII were not available such as eugenol, ginger, $n-3$, and $n-6$ fatty acids, saffron, trans fat, turmeric, thyme/oregano, rosemary, and flavonoids, which may have influenced our results.

In conclusion, our findings suggest that a higher DII score was associated with an increased risk of DSPN. The results remained significant after adjustment for covariates. Therefore, it is possible that a diet rich in anti-inflammatory nutrients and foods could improve and prevent DSPN. However, trial studies are required to clarify the effect of DII on DSPN.

\section{Declarations}

\section{Acknowledgments}

This research was supported financially by the Tehran University of Medical Sciences. This study was conducted in the Diabetes Research Center, Endocrinology and Metabolism Clinical Sciences Institute, Kermanshah University of Medical Sciences, Kermanshah, Iran. We thank the authorities who help us and all patients who participated in this research. All authors declare no personal or financial conflicts of interest

\section{References}

1. Organization, W.H., Classification of diabetes mellitus. 2019.

2. Callaghan, B.C., et al., Diabetic neuropathy: the future is promising. Reply to Uusitupa M, Niskanen L, Laitinen T [letter] and Coppini DV. Diabetologia, 2020: p. 1-2. 
3. Girach, A., et al., Quality of life in painful peripheral neuropathies: a systematic review. Pain Research and Management, 2019. 2019.

4. Gylfadottir, S.S., et al., Painful and non-painful diabetic polyneuropathy: Clinical characteristics and diagnostic issues. Journal of diabetes investigation, 2019. 10(5): p. 1148-1157.

5. Halim, M. and A. Halim, The effects of inflammation, aging and oxidative stress on the pathogenesis of diabetes mellitus (type 2 diabetes). Diabetes \& Metabolic Syndrome: Clinical Research \& Reviews, 2019. 13(2): p. 1165-1172.

6. Zilliox, L.A. and J.W. Russell, Physical activity and dietary interventions in diabetic neuropathy: a systemic review. Clinical Autonomic Research, 2019: p. 1-13.

7. Arouca, A.B., et al., Diet as a moderator in the association of sedentary behaviors with inflammatory biomarkers among adolescents in the HELENA study. European journal of nutrition, 2019. 58(5): p. 2051-2065.

8. Carvalho, C.A., et al., The dietary inflammatory index and insulin resistance or metabolic syndrome in young adults. Nutrition, 2019. 58: p. 187-193.

9. Arabzadegan, N., et al., Effects of dietary whole grain, fruit, and vegetables on weight and inflammatory biomarkers in overweight and obese women. Eating and Weight Disorders-Studies on Anorexia, Bulimia and Obesity, 2019: p. 1-9.

10. Shivappa, N., et al., Designing and developing a literature-derived, population-based dietary inflammatory index. Public health nutrition, 2014. 17(8): p. 1689-1696.

11. Shivappa, N., et al., Association between dietary inflammatory index and inflammatory markers in the HELENA study. Molecular nutrition \& food research, 2017. 61(6): p. 1600707.

12. Shivappa, N., et al., A population-based dietary inflammatory index predicts levels of C-reactive protein in the Seasonal Variation of Blood Cholesterol Study (SEASONS). Public health nutrition, 2014. 17(8): p. 1825-1833.

13. Schulze, M.B., et al., Dietary pattern, inflammation, and incidence of type 2 diabetes in women-. The American journal of clinical nutrition, 2005. 82(3): p. 675-684.

14. Denova-Gutiérrez, E., et al., Dietary inflammatory index and type 2 diabetes mellitus in adults: the diabetes mellitus survey of Mexico City. nutrients, 2018. 10(4): p. 385.

15. Moslehi, N., et al., Inflammatory properties of diet and glucose-insulin homeostasis in a cohort of Iranian adults. Nutrients, 2016. 8(11): p. 735.

16. Vahid, F., et al., Association between Dietary Inflammatory Index (DII) and risk of prediabetes: a casecontrol study. Applied Physiology, Nutrition, and Metabolism, 2017. 42(4): p. 399-404.

17. Esfahani, F.H., et al., Reproducibility and relative validity of food group intake in a food frequency questionnaire developed for the Tehran Lipid and Glucose Study. Journal of epidemiology, 2010. 20(2): p. 150-158.

18. Monteiro, C.A., et al., Increasing consumption of ultra-processed foods and likely impact on human health: evidence from Brazil. Public health nutrition, 2010. 14(1): p. 5-13. 
19. Bostani, A. and H. HOMAYOUNFAR, The Relationship between NCS Findings and Toronto Clinical Scoring System of Neuropathy in Diabetic Polyneuropathy. 2006.

20. Bril, V. and B.A. Perkins, Validation of the Toronto Clinical Scoring System for diabetic polyneuropathy. Diabetes Care, 2002. 25(11): p. 2048-2052.

21. Aadahl, M. and T. Jørgensen, Validation of a new self-report instrument for measuring physical activity. Medicine and science in sports and exercise, 2003. 35(7): p. 1196-1202.

22. Bunner, A., et al., A dietary intervention for chronic diabetic neuropathy pain: a randomized controlled pilot study. Nutrition \& diabetes, 2015. 5(5): p. e158-e158.

23. Kellogg, A.P., et al., Protective effects of cyclooxygenase-2 gene inactivation against peripheral nerve dysfunction and intraepidermal nerve fiber loss in experimental diabetes. Diabetes, 2007. 56(12): p. 2997-3005.

24. Wang, Y., et al., Enhanced inflammatory response via activation of NF-KB in acute experimental diabetic neuropathy subjected to ischemia-reperfusion injury. Journal of the neurological sciences, 2006. 247(1): p. 47-52.

25. Giugliano, D., A. Ceriello, and K. Esposito, The effects of diet on inflammation: emphasis on the metabolic syndrome. Journal of the American College of Cardiology, 2006. 48(4): p. 677-685.

26. Cavicchia, P.P., et al., A new dietary inflammatory index predicts interval changes in serum highsensitivity C-reactive protein. The Journal of nutrition, 2009. 139(12): p. 2365-2372.

27. Huang, W.-Q., et al., A higher Dietary Inflammatory Index score is associated with a higher risk of breast cancer among Chinese women: a case-control study. British Journal of Nutrition, 2017. 117(10): p. 1358-1367.

28. Pop-Busui, R., et al., Inflammation as a therapeutic target for diabetic neuropathies. Current diabetes reports, 2016. 16(3): p. 29.

29. Edwards, J.L., et al., Diabetic neuropathy: mechanisms to management. Pharmacology \& therapeutics, 2008. 120(1): p. 1-34.

30. Harati, Y., Diabetic peripheral neuropathies. Annals of internal medicine, 1987. 107(4): p. 546-559. 